ORIGINAL RESEARCH

\title{
Nonintrusive Biological Signal Monitoring in a Car to Evaluate a Driver's Stress and Health State
}

Hyun Jae Baek, M.S., ${ }^{1}$ Haet Bit Lee, M.S., ${ }^{1}$ Jung Soo Kim, Ph.D., ${ }^{2}$ Jong Min Choi, Ph.D., ${ }^{3}$ Ko Keun Kim, Ph.Dc., ${ }^{2}$ and Kwang Suk Park, Ph.D.

${ }^{1}$ Graduate Program in Bioengineering, Seoul National University, Seoul, Korea.

${ }^{2}$ Institute of Medical and Biological Engineering, Medical Research Center, Seoul National University, Seoul, Korea.

${ }^{3}$ Research Center for Sensory Organ, Medical Research Center, Seoul National University, Seoul, Korea.

${ }^{4}$ Department of Biomedical Engineering, College of Medicine, Seoul National University, Seoul, Korea.

\section{Abstract}

Nonintrusive monitoring of a driver's physiological signals was introduced and evaluated in a car as a test of extending the concept of ubiquitous healthcare to vehicles. Electrocardiogram, photoplethysmogram, galvanic skin response, and respiration were measured in the ubiquitous healthcare car (U-car) using nonintrusively installed sensors on the steering wheel, driver's seat, and seat belt. Measured signals were transmitted to the embedded computer via Bluetooth ${ }^{\circledR}$ communication and processed. We collected and analyzed physiological signals during driving in order to estimate a driver's stress state while using this system. In order to compare the effect of stress on physical and mental conditions, two categories of stresses were defined. Experimental results show that a driver's physiological signals were measured with acceptable quality for analysis without interrupting driving, and they were changed meaningfully due to elicited stress. This nonintrusive monitoring can be used to evaluate a driver's state of health and stress.
Key words: nonintrusive, $u$-healthcare, $u$-car, ECG, PPG, respiration, stress, vehicle

\section{Introduction}

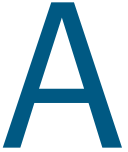

$s$ the average life expectancy has increased, the amount of attention paid to healthcare services outside of hospitals has increased. Vital signs that reflect health status are relatively easy to measure. Early devices for biosignal measurement required additional instruments, lead wires for operating devices, and electrodes that must be attached to the skin. Even though inconvenience to the user is sometimes acceptable, vehicular measurement was not practical because it requires the user's attention and cooperation.

As healthcare technology has developed, nonintrusive biosignal measurement techniques have been widely studied and developed. ${ }^{1,2}$ Most systems for ubiquitous healthcare have concentrated on homebased biosignal monitoring. In these studies, sensors are usually attached to an appliance such as a bed, toilet seat, or couch. ${ }^{3-6}$ These kinds of systems provide fully unobtrusive healthcare information. All measurements are made without the user's awareness. However, ubiquitous healthcare has not been extended beyond the home environment.

In recent years, cars have become more common in industrialized societies. Many people spend much of their time in a car. Therefore, to further develop the ubiquitous healthcare concept, health monitoring during driving is needed.

The ubiquitous healthcare car that we suggest in this study is hereafter called the "U-car." This study extends the ubiquitous healthcare system to a vehicle. A U-car that can monitor a driver's physiological signals nonintrusively was introduced, and its performance was evaluated. 


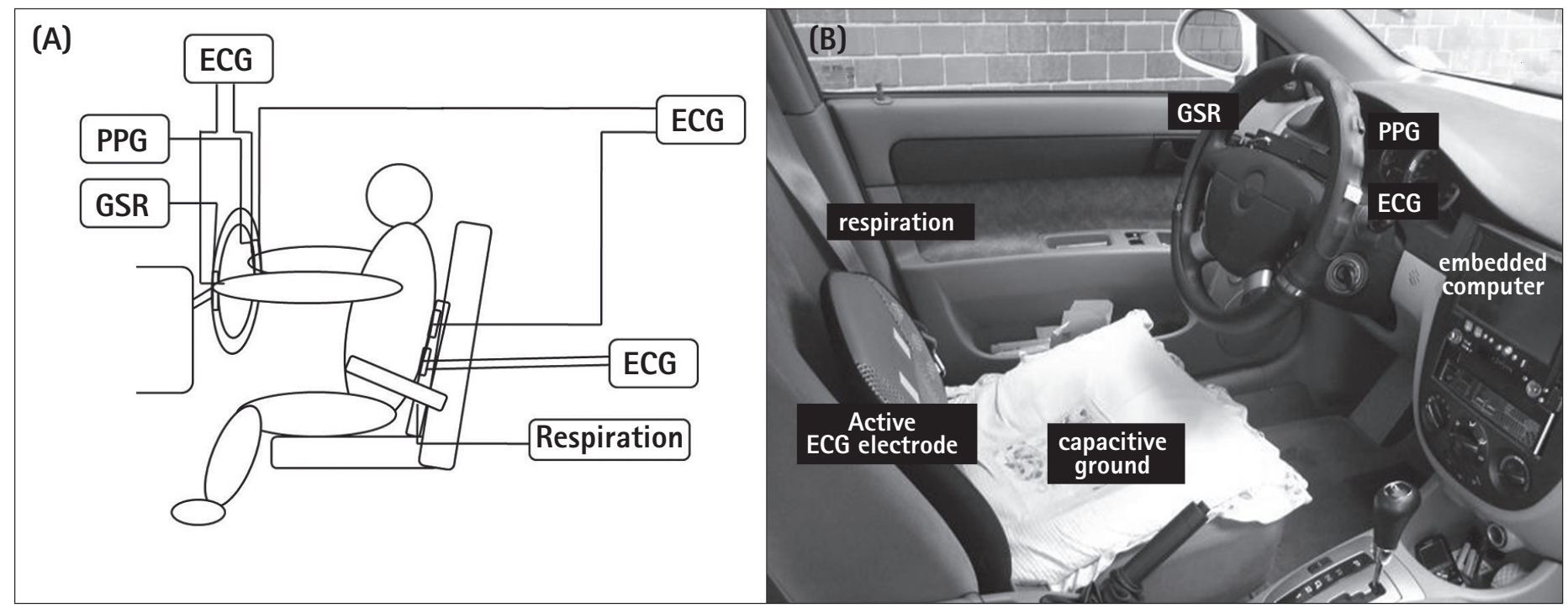

Fig. 1. Sensors of the U-car system and their locations. (A) Setup of the physiological signal acquisition system in the car. GSR, galvanic skin response; PPG, photoplethysmogram; ECG, electrocardiogram. (B) Settings in a real car with installed sensors.

\section{Materials and Methods}

\section{SYSTEM ARCHITECTURE}

The schema of the proposed system is shown in Figure 1. For nonintrusive measurement of a driver's biosignals, sensors were attached to the steering wheel, driver's seat, and seat belt.

The copper tapes that were attached to the steering wheel were used for measuring electrocardiogram (ECG) and galvanic skin response (GSR) from subjects' palms. A reflective-type photoplethysmogram (PPG) sensor (NONIN finger clip 8000K2, USA) was also placed on the steering wheel. Capacitive coupled electrodes for ECG measurement, which were applied previously on a chair by our group, ${ }^{4,5}$ were adapted to the car. Because the capacitance between the electrode and the driver's back is not high, a high-input impedance preamplifier that converts current into voltage was mounted on the back of each active electrode. As a capacitive ground, a conductive textile was laid on the driver seat. The combination of a capacitive coupled ECG electrode and copper tape was attempted by using an active electrode attached to the backrest of the driver seat, and copper tape was placed on the steering wheel. A piezoelectric respiration transducer (Pneumotrace, MorroBay, CA) was attached to the seat belt. The summary of the sensors used in the U-car is shown in Table 1.

All measured signals were converted to a digital signal at a $200-\mathrm{Hz}$ sampling rate and a resolution of 10 bits. Digitized signals were then transmitted to the embedded computer in the U-car via Bluetooth ${ }^{\circledR}$ (Motorola, Inc., Schaumburg, IL) wireless communication. The signal viewer program was developed with Visual Studio 2005 (Microsoft, Redmond, WA) and TeeChart pro (SteeMa Software, Spain). In the viewer program, PPG, GSR, ECGs, and respiration signals are displayed in order. The first ECG was measured from the driver's palms and the second one was from the driver's back. The last one was measured from the driver's palm and back. An example of measured signals is illustrated in Figure 2.

\begin{tabular}{l|l|l}
\multicolumn{2}{l}{$\begin{array}{l}\text { Table 1. Location of Environmental Sensors of U-Car } \\
\text { LOCATION }\end{array}$} & \multicolumn{1}{c}{ MATERIAL } \\
\multirow{2}{*}{ Steering wheel } & Copper sheet & \multicolumn{1}{c}{ TARGET } \\
\cline { 2 - 3 } & Copper sheet & Galvanic skin response \\
\cline { 2 - 3 } & LED and photodiodes & Photoplethysmogram \\
\hline Driver seat & Capacitive coupled electrode & Electrocardiogram \\
\hline Seatbelt & Piezoelectric & Respiration \\
\hline $\begin{array}{l}\text { Steering wheel and } \\
\text { driver seat }\end{array}$ & $\begin{array}{l}\text { Copper sheet, Capacitive } \\
\text { coupled electrode }\end{array}$ & Electrocardiogram \\
\hline
\end{tabular}

LED, light-emitting diode. 
BAEK ET AL.

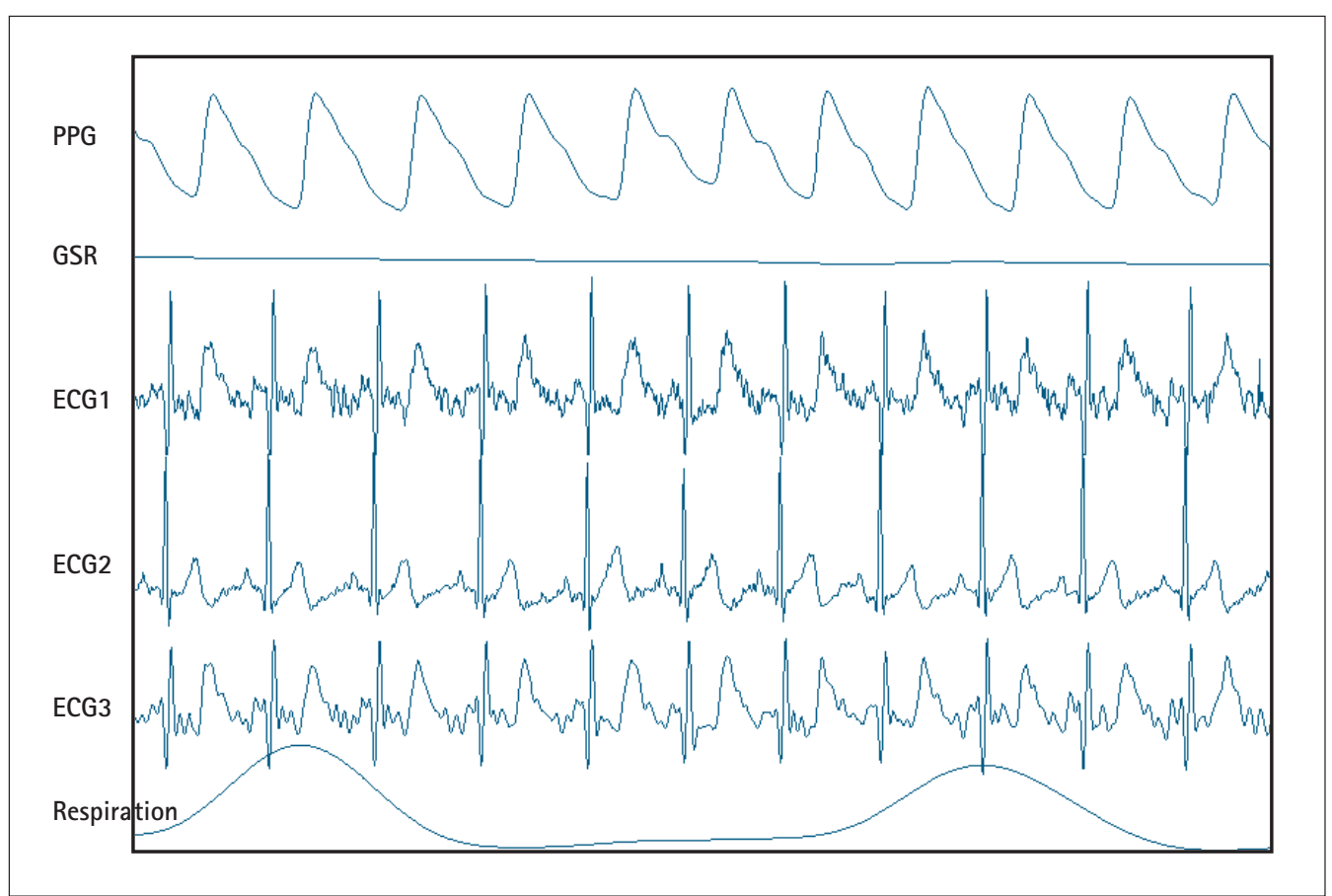

Fig. 2. Example of measured driver's biological signals. PPG, photoplethysmogram; GSR, galvanic skin response; ECG, electrocardiogram.

first derivative point of PPG, and the respiratory rate $(\mathrm{RR})$ were compared for validation of ECG, PPG, and respiration measured using the U-car system, respectively.

\section{Stress elicitation.}

There are many different definitions of stress. ${ }^{7,14}$ According to Esch, stress is interpreted as a general term that describes the effects of psychosocial and environmental factors on physical or mental wellbeing. ${ }^{14,15}$ From this point of view, each stressor was classified into two categories in this study. The method for the elicitation of stress was to create a situation similar to real load driving. In order to compare the effect of stress on the driver's body condition, driving in cold and hot environments and night driving were employed as physical stresses (Table 2). The temperature inside the car was fixed at $13^{\circ} \mathrm{C}$ in the cold environment experiment and $40^{\circ} \mathrm{C}$

\section{REAL-LOAD DRIVING}

The study included four volunteers (males, age range 25-31 years) with a minimum of 5 years of driving experience. They had no history of cardiovascular-related diseases. All subjects practiced driving before the experiment to familiarize themselves with the U-car. In the experiments, each subject performed the driving experiment twice.

Stress can introduce unexpected diseases and gradually make subjects have chronic fatigue. ${ }^{7-9}$ Many traffic accidents are caused by driver inattention and are due to the driver's stress. Therefore, evaluating a driver's level of stress is important to maintaining a driver's health and to preventing traffic accidents. Biosignals reflect the driver's physiological condition. ${ }^{10-13}$ For these reasons, nonintrusively monitored signals were analyzed simultaneously with the subjective stress scale of drivers to investigate the relationship between them.

Validation of U-car system.

A conventional physiological signal acquisition system (MP150, Biopac, USA) was used to measure reference ECG, PPG, and respiration for comparison purposes. R-peak interval time, the interval of in the hot environment experiment. The biosignals measured in each condition were compared with those of the driver's normal environmental state when the temperature was about $25^{\circ} \mathrm{C}$. Night driving was compared with daytime driving. Second, arithmetic operation, driving in a noisy environment, and driving in a dark alley were selected as mental stresses. Subjects were asked to calculate simple arithmetic operations as quickly as possible during driving. The four fundamental rules of arithmetic were employed for this task. It consisted of maximally two-digit random numbers that were generated by a computer (e.g., $25 \times 3$ ). The researcher asked the problem and subjects were instructed to say the answer loudly as quickly as possible.

Table 2. Stress Elicitation Methods

PHYSICAL STRESS

MENTAL STRESS

\begin{tabular}{l|l} 
Night driving (NIGHT) & Arithmetic operation (MATH) \\
\hline Driving in cold environment (COLD) & Driving in noisy environment (NOISY) \\
\hline Driving in hot environment (HOT) & Driving in a narrow alley (NARROW) \\
\hline
\end{tabular}


If they made any mistakes, they were quickly warned, and continued the task as the experimenter instructed. Classic and rock music were employed as noise sources. Driving in a narrow alley increases mental tension in the driver. All of these contribute to driver stress.

\section{Experimental methods.}

The subjects drove the U-car in the city of Seoul. Before stress elicitation, each driver's biosignals were recorded for 6 minutes in a resting state. Then 6 minutes of recording were performed with elicited stress. To eliminate interference between stress elicitations, recovery driving was performed for 15 minutes between stress elicitations.

\section{Subjective stress score.}

At the end of each stress condition, a subjective stress level weighted from 0 to 10 was reported to compare changes in the driver's biosignals. The drivers marked stress level by subjective personal judgment. After all stress conditions, drivers reviewed their answer about each stress condition and rescored according to comparative stress level, where 10 was the highest stress and 0 was the lowest stress. Although there is no gold standard self-report evaluation of stress, subjective stress score that was employed in this study is enough to show comparable stress level with personally perceived stress. known that HRV is related to sympathetic and parasympathetic activity of the autonomic nervous system. Blood pressure is also related to them, and it has an inverse correlation with pulse arrival time (PAT). PAT is the time interval between the ECG R-peak and the characteristic point of the pulse wave of the peripheral artery. In this study, the first derivative point of PPG was used to calculate PAT.

An example of an analyzed signal is shown in Figures 3, 4, and 5. Based on the dotted line, the left side of the signal represents resting state, and the right side shows elicited stress conditions. When drivers were subjected to a stress stimulus, changes in parameters showed larger variation than in the resting state.

In order to compare variation in parameters, each parameter was calculated as described below. The average value of a parameter in a stress condition was compared with that of the resting state. A decrease in parameter value was represented as "-" and an increase in parameter value as "+". Table 3 shows the direction of parameter change for each stress condition. Although the respiratory rate showed less significant changes than other factors, almost all data showed significant results, especially R-peak interval time. The specific quantity of changes in HRV parameters (heart rate, standard deviation of all NN intervals, square root of the mean of the sum of the squares of differences between adjacent NN interval, percent

\section{Results}

\section{TEST FOR EVALUATION OF U-CAR}

The analysis result for validation between two measurement systems shows very high correlation. The correlation coefficients for ECG, PPG, and respiration were 0.986, 0.970, and 0.965, respectively. A Bland-Altman plot also presents considerable correlation between the two measurement systems. From the validation results, the U-car system is considered to measure biological signals as accurately as conventional reference signal measurement methods.

\section{STRESS-RELATED BIOSIGNAL ANALYSIS}

From the driver's measured physiological signals, R-peak interval time, pulse arrival time, and respiratory rate were calculated. Heart rate variability (HRV) is computed based on R-peak. It is well

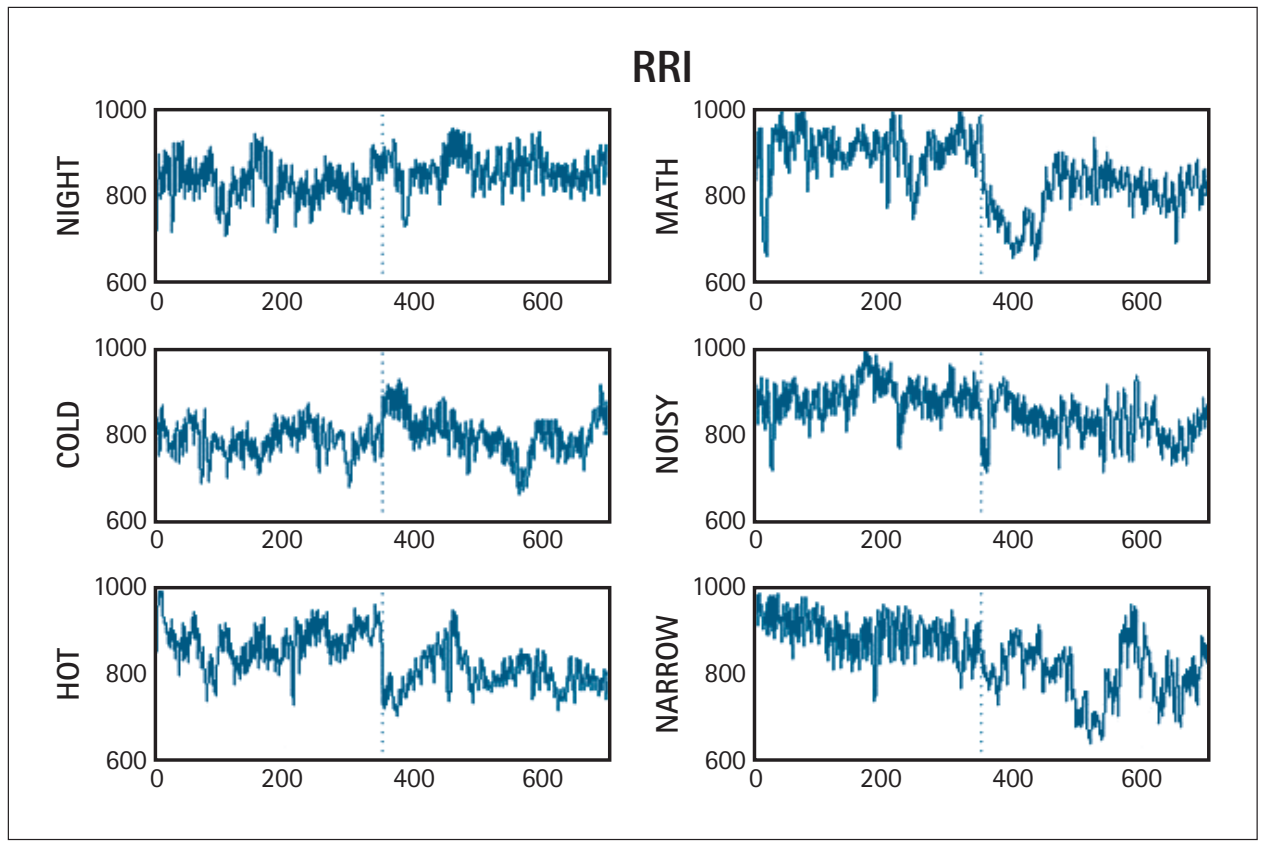

Fig. 3. Changes in R-peak interval time. The resting state and stress states are distinguished by a dotted line (left side: resting state, right side: stress state). RRI, R-peak interval time. 
BAEK ET AL.

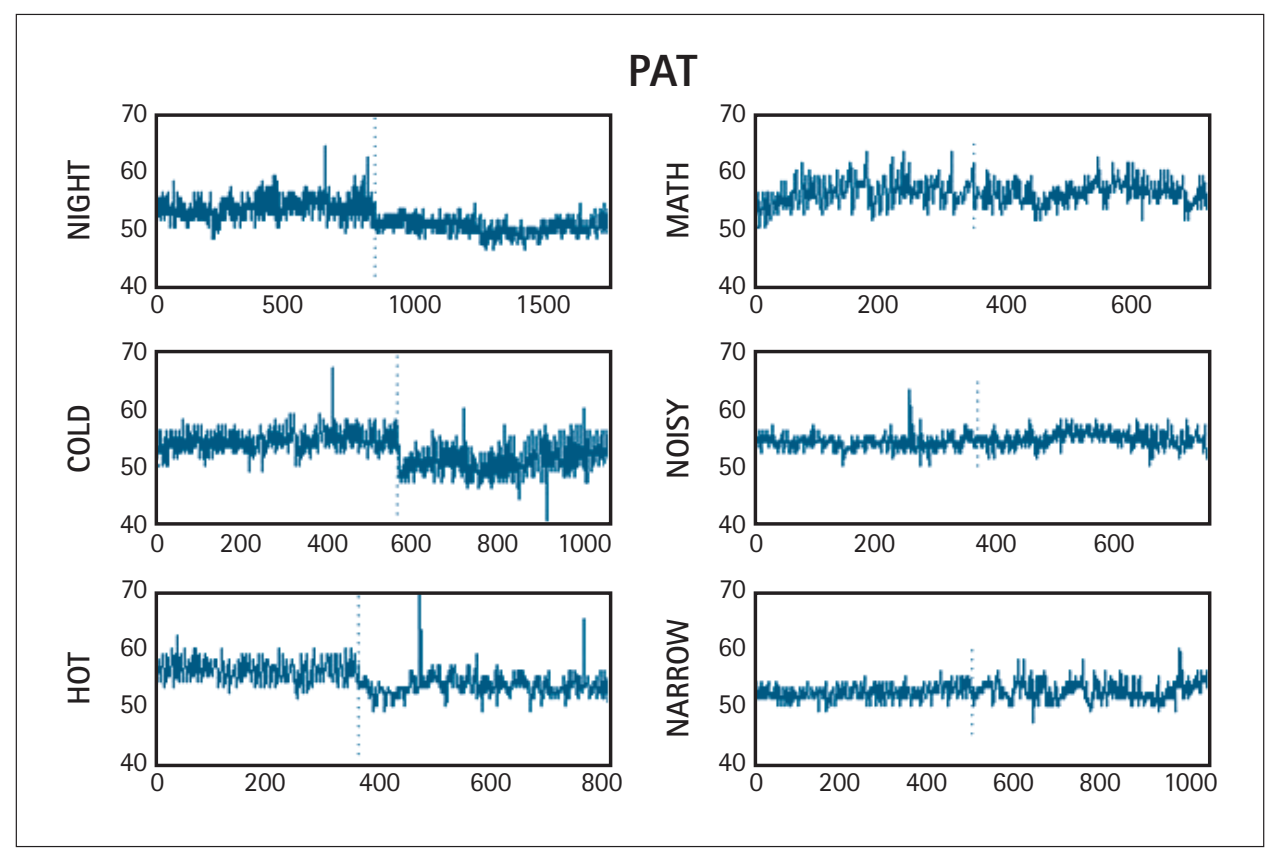

Fig. 4. Changes in pulse arrival time (PAT). The resting state and stress states are distinguished by a dotted line (left side: resting state, right side: stress state).

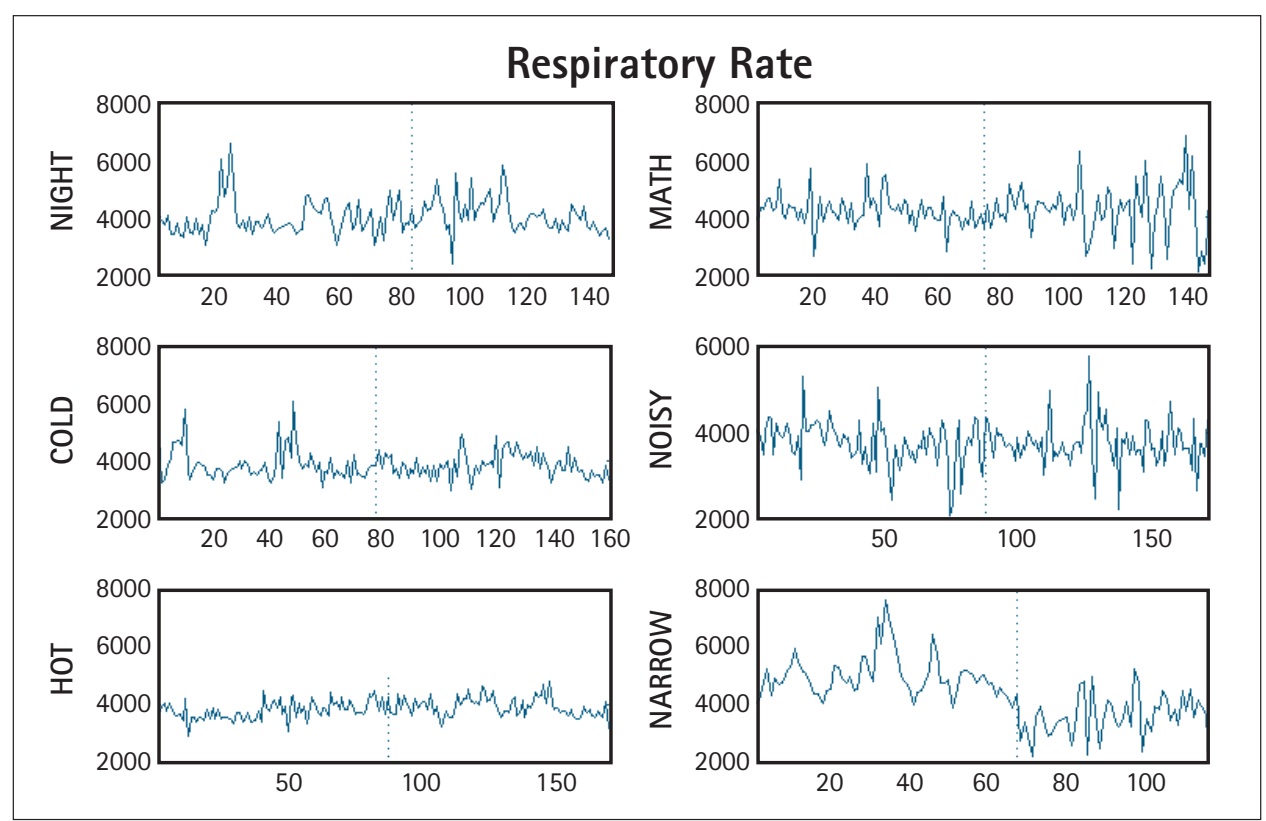

Fig. 5. Changes in the respiratory rate. The resting state and stress states are distinguished by dotted line (left side: resting state, right side: stress state). of difference between adjacent NN intervals that are greater than $50 \mathrm{~ms}$, ratio of low-high frequency power, low-frequency power, high-frequency power), PAT, and respiratory rate was not mentioned here, but in most cases, HRV parameters showed large changes, especially in the physical stress condition.

As for the average driver's response on the subjective stress scale, driving in a cold environment and arithmetic operation were highest among the physical stress and mental stress factors, respectively. Elicited stresses are presented in Table 4, in the order of strength. Time domain HRV parameters showed the best potential for detecting a driver's stress during normal driving.

\section{Discussion}

\section{REAL CAR EXPERIMENT WITH} NONINTRUSIVE MONITORING OF

\section{DRIVER'S BIOSIGNALS}

In the driver's biosignal research area, adhesive electrodes have usually been employed to acquire physiological signals. ${ }^{16,17}$ They obstruct the driver's behavior with wires that connect sensors to the acquisition device. The U-car system employs environmental sensors. Therefore, a driver's physiological signals can be acquired without disturbing the driver's behavior. A virtual reality-based driving simulator has been used in many driverrelated studies. ${ }^{11,17,18}$ Although this kind of laboratory system makes it easy to control the environment of the experiment and to reduce the effect of noise, it does not reflect realistic traffic conditions for daily health monitoring. From this point of view, stressrelated experiments in a driving simulator are not comparable to actual stress. Because subjects already know that the experimental environment is not real driving but virtual reality, they may not feel normal levels of tension. When there is no real disadvantage 


\begin{tabular}{|c|c|c|c|c|c|c|c|}
\hline & \multirow{2}{*}{ SUBJECT } & \multicolumn{3}{|c|}{ PHYSICAL STRESS } & \multicolumn{3}{|c|}{ MENTAL STRESS } \\
\hline & & NIGHT & COLD & HOT & MATH & NOISY & NARROW \\
\hline \multirow{8}{*}{ RRI } & $A-1$ & $-^{* *}$ & $+^{* *}$ & ns & $-^{* *}$ & ns & $-^{* *}$ \\
\hline & $\mathrm{A}-2$ & $-^{* *}$ & $+^{* *}$ & $-* *$ & $-^{* *}$ & $-^{* *}$ & $-^{* *}$ \\
\hline & B-1 & $-^{* *}$ & $+^{* *}$ & ns & ns & $t^{* *}$ & $-^{* *}$ \\
\hline & B-2 & $-^{* *}$ & $+^{* *}$ & $-^{* *}$ & $-^{* *}$ & $-^{* *}$ & $-^{* *}$ \\
\hline & $\mathrm{C}-1$ & $t^{* *}$ & $t^{* *}$ & $--^{* *}$ & $-{ }_{-* *}^{* *}$ & $-^{* *}$ & $--^{* *}$ \\
\hline & $C-2$ & $+^{* *}$ & $-^{* *}$ & $--^{* *}$ & $--^{* *}$ & $+^{* *}$ & $--^{* *}$ \\
\hline & D-1 & $+^{* *}$ & $t^{* *}$ & $--^{* *}$ & $+^{* *}$ & $--^{* *}$ & $--^{* *}$ \\
\hline & D-2 & $-^{* *}$ & $t^{* *}$ & $-2^{* *}$ & $t^{* *}$ & $-^{* *}$ & $-* *$ \\
\hline \multirow{8}{*}{ PAT } & $A-1$ & $-^{* *}$ & $-^{* *}$ & $-^{* *}$ & - & $+^{* *}$ & $+^{* *}$ \\
\hline & $\mathrm{A}-2$ & $-^{* *}$ & $-^{* *}$ & $t^{*}$ & $-{ }_{-*}^{* *}$ & $+^{* *}$ & - \\
\hline & B-1 & $++^{* *}$ & $-^{* *}$ & $-{ }^{* *}$ & - & $t^{* *}$ & $-^{* *}$ \\
\hline & B-2 & $--^{* *}$ & $-^{* *}$ & -** & - $^{* *}$ & $-^{* *}$ & $+^{* *}$ \\
\hline & $C-1$ & - & $-^{* *}$ & $t^{* *}$ & $-^{* *}$ & $-2^{* *}$ & $--^{* *}$ \\
\hline & $\mathrm{C}-2$ & $-^{* *}$ & $-z^{* *}$ & - & $-_{-*}^{* *}$ & - $^{*}$ & -** \\
\hline & D-1 & $-^{* *}$ & $-^{* *}$ & + & + & $+^{* *}$ & -** $^{* *}$ \\
\hline & D-2 & - & $t^{* *}$ & $+^{* *}$ & -** $^{* *}$ & $-^{* *}$ & - $^{* *}$ \\
\hline \multirow{8}{*}{$\mathrm{RR}$} & $A-1$ & + & - & - & $-^{* *}$ & $+^{* *}$ & $-^{* *}$ \\
\hline & $\mathrm{A}-2$ & + & $+^{* *}$ & $+^{* *}$ & - $^{* *}$ & - & $-^{* *}$ \\
\hline & B-1 & + & - & $+^{*}$ & $+^{*}$ & - & $-^{* *}$ \\
\hline & B-2 & - & $t^{* *}$ & - & + & - & - \\
\hline & $C-1$ & - & - & $+^{* *}$ & - & - & $-^{* *}$ \\
\hline & $C-2$ & - & + & $-^{* *}$ & + & + & $-^{* *}$ \\
\hline & D-1 & $-^{*}$ & - & + & - & - & + \\
\hline & D-2 & ns & + & - & - & - & + \\
\hline
\end{tabular}

${ }^{*} p<0.05,{ }^{* *} p<0.001$.

or harm to the subject for an accident or mistake in virtual reality, the subject may not feel real stress. Therefore, this work, which includes real driving experiments, is a better method of studying the relationship between stress and relative changes in physiological signals.

\section{APPROACH FOR THE ESTIMATION OF A DRIVER'S STRESS STATE}

In this study, one remarkable aspect of the experimental results is that different reactions were observed for the same stressor. This phenomenon can be explained as individual differences in the stress response. Stress is a general term that describes a subject's state and that has an influence on the subject's psychophysical reaction. In other words, although the same stressor was given to subjects, their responses varied. A stressor employed in this study could cause serious stress for some subjects, but not for others. Similarly, the stress response of the same subject varied between experiments in some cases. It can be explained as a temporal difference. We conclude that the irregular direction in the changes in physiological signals was 


\begin{tabular}{|c|c|c|c|c|c|c|}
\hline \multirow[t]{2}{*}{ PARAMETER } & \multicolumn{3}{|c|}{ PHYSICAL STRESS } & \multicolumn{3}{|c|}{ MENTAL STRESS } \\
\hline & $1 s t$ & 2nd & $3 r d$ & $1 s t$ & 2nd & $3 r d$ \\
\hline RR & COLD & NIGHT & НОТ & MATH & NOISY & NARROW \\
\hline PAT & $\mathrm{NIGHT}$ & COLD & НОT & MATH & NOISY & NARROW \\
\hline $\mathrm{HR}$ & HOT & NIGHT & COLD & NOISY & MATH & NARROW \\
\hline SDNN & COLD & NIGHT & НОТ & MATH & NOISY & NARROW \\
\hline RMSSD & COLD & NIGHT & НОТ & NOISY & MATH & NARROW \\
\hline pNN50 & COLD & NIGHT & HOT & NOISY & NARROW & MATH \\
\hline LF/HF & HOT & COLD & NIGHT & MATH & NARROW & NOISY \\
\hline LF & HOT & COLD & NIGHT & NARROW & MATH & NOISY \\
\hline $\mathrm{HF}$ & HOT & COLD & NIGHT & NARROW & NOISY & MATH \\
\hline Stress score & COLD & HOT & NIGHT & NOISY & MATH & NARROW \\
\hline
\end{tabular}

RR, Respiratory rate; PAT, Pulse arrival time; HR, Heart rate; SDNN, Standard deviation of all NN intervals; RMSSD, Square root of the mean of the sum of the squares of differences between adjacent NN interval; pNN50, Percent of difference between adjacent NN intervals that are greater than 50 ms; LF/HF, Ratio of low-high-frequency power; LF, Low-frequency power $(0.04-0.15 \mathrm{~Hz})$; HF, High-frequency power $(0.15-0.4 \mathrm{~Hz})$.

due to individual and temporal differences. The most meaningful parameter that showed changes due to stressors was revealed to be the time domain parameters of HRV by statistical analysis and t-test. The frequency domain parameters of HRV are more efficient with slow changes. On the other hand, the time domain parameters of HRV reflect instant changes well. Because stress that occurs during driving can occur instantly, the HRV time domain parameter was efficient in this experiment. In conclusion, physiological signals were changed meaningfully during stimulated driving. A combination of physiological parameters has the potential to evaluate a driver's state.

\section{CLINICAL APPLICATION FOR TELEMEDICINE}

Driving requires sustained attention on continual interaction of intrinsic and extrinsic factors. This attention may be affected by the driver's health status and stress. Therefore, health monitoring of the driver and a related feedback system are essential for safe driving.

The U-car system includes cardiovascular-related sensors; therefore, a high-risk patient can be monitored. When untoward events associated with cardiovascular-related disease occur during driving the U-car, the driver can take whatever action is necessary to rectify the situation through immediate user feedback. Furthermore, through remote monitoring a doctor can treat the driver until he arrives at the hospital.

In summary, a method utilizing biological signals can be provided to ensure safe driving in an abnormal situation.

\section{LIMITATION}

When a subject handled the steering wheel, a motion artifact or intermittent signal loss occurred. To obtain clear signals, subjects must grip the steering wheel during all driving. Some signal loss is acceptable because continuous signal recording is not necessary for health monitoring. Also, in the case of the electrocardiogram, motion artifact and signal loss can be compensated for by using secondary acquisition through the driver seat.

We have two experiments for each subject in this study, but more experiments are needed to study individual differences in the stress response. The combination of physical and mental stress variable condition will be considered for assessment of a real-world situation in future research.

\section{Conclusions}

A nonintrusive system for monitoring a driver's health and its application for estimation of a driver's stress was proposed. So far, most systems for ubiquitous healthcare have focused on homebased systems with sensors attached to home appliances. In this work, the ubiquitous healthcare concept is extended to the vehicle by employing sensors placed on the steering wheel, driver seat, and seatbelt. Experimental results showed that a driver's biosignal could be measured nonintrusively, and it was changed according to the driver's stress state. Based on the present work, long-term daily health monitoring is possible not only in the home but also in the car. 


\section{BIOLOGICAL SIGNAL MONITORING IN CAR TO EVALUATE DRIVER'S STATE}

Stress-induced traffic accidents may be prevented by user feedback of detected changes in parameters extracted from a driver's physiological signals.

\section{Acknowledgments}

This study was supported by a grant from the Advanced Biometric Research Center (ABRC), the Korea Science and Engineering Foundation (KOSEF), and the Ministry of Information and Communication (MIC), under the Information Technology Research Center (ITRC) support program.

\section{Disclosure Statement}

No competing financial interests exist.

\section{REFERENCES}

1. Lymberis A, Olsson S. Intelligent biomedical clothing for personal health and disease management: State of the art and future vision. Telemed E-Health 2003;9:379-386

2. Seo J, Choi J, Choi B, Jeong DU, Park K. The development of a nonintrusive home-based physiologic signal measurement system. Telemed J E-Health 2005;11:487-495.

3. Kim JS, Chee YJ, Park JW, Choi JW, Park KS. A new approach for non-intrusive monitoring of blood pressure on a toilet seat. Physiol Meas 2006;27:203-211.

4. Kim J, Park J, Kim J, Chee Y, Lim Y, Park K. Development of a nonintrusive blood pressure estimation system for computer users. Telemed J E-Health 2007;13:57-64.

5. Lim YG, Kim KK, Park KS. ECG measurement on a chair without conductive contact. IEEE Trans Biomed Eng 2006;53:956-959.

6. Lim YG, Kim KK, Park KS. ECC recording on a bed during steep without direct skin-contact. IEEE Trans Biomed Eng 2007;54:718-725.

7. Hubbard TE Jr. Handbook of stress medicine: An organ system approach. Boca Raton, FL: CRC Press, 1998.

8. Babisch W, Beule B, Schust M, Kersten N, Ising H. Traffic noise and risk of myocardial infarction. Epidemiology 2005;16:33-40.

9. Esch T, Stefano GB, Fricchione GL, Benson H. Stress in cardiovascular diseases. Med Sci Monitor 2002;8:RA93-RA101.
10. Polanczyk A, Rohde LE, Moraes RS, Ferlin EL, Leite C, Ribeiro JP. Sympathetic nervous system representation in time and frequency domain indices of heart rate variability. Eur J Appl Physiol Occup Physiol 1998;79:69-73.

11. Jiao K, Li ZY, Chen M, Wang CT, Qi SH. Effect of different vibration frequencies on heart rate variability and driving fatigue in healthy drivers. Int Arch Occup Envir Health 2004;77:205-212.

12. Hjortskov N, Rissen D, Blangsted AK, Fallentin N, Lundberg U, Sogaard K. The effect of mental stress on heart rate variability and blood pressure during computer work. Eur J Appl Physiol 2004;92:84-89.

13. Shusterman V, Barnea 0 . Sympathetic nervous system activity in stress and biofeedback relaxation. IEEE Eng Med Biol Mag 2005;24:52-57.

14. Selye $H$. The evolution of the stress concept: Stress and cardiovascular disease. Am J Cardiol 1970;26:289-299.

15. Esch T. Gesund im stress: Der wandel des stresskonzeptes und seine bedeutung fur pravention, gesundheit und lebensstil [Health in stress: Change in the stress concept and its significance for prevention, health and life style]. Gesundheitswesen 2002;64:73-81.

16. Healey JA, Picard RW. Detecting stress during real-world driving tasks using physiological sensors. IEEE Trans Intelligent Transport Syst 2005;6:156-166.

17. Tejero P, Choliz M. Driving on the motorway: The effect of alternating speed on driver's activation level and mental effort. Ergonomics 2002;45:605-618.

18. Ji Q, Zhu ZW, Lan PL. Real-time nonintrusive monitoring and prediction of driver fatigue. IEEE Trans Vehicular Techno/ 2004;53:1052-1068.

Address reprint requests to: Kwang Suk Park, Ph.D. Department of Biomedical Engineering College of Medicine Seoul National University Yongon-Dong, Jongno-Gu

Seoul, 110-799

Republic of Korea

E-mail: kspark@bmsil.snu.ac.kr

Received: June 24, 2008 Accepted: August 5, 2008 